\title{
Desarrollo de una propuesta de estándar de calidad en la línea de calzado escolar
}

\author{
Alba Yaneth Góchez Martínez ${ }^{1}$ \\ Jasmín Rocío Retana de Alemán² \\ Alfredo de Jesús Grande Sánchez ${ }^{3}$ \\ Docentes investigadores, Facultad de Ingeniería y Arquitectura \\ Universidad Católica de El Salvador, El Salvador
}

Fecha de recepción: 16-12-2019

Fecha de aceptación: 21-02-2020

\section{Resumen}

Las empresas de la industria del calzado en El Salvador, particularmente las micro, pequeñas y medianas, enfrentan situaciones que les restan competitividad como: los problemas de calidad de las materias primas (cuero), componentes (suelas, cordones, plantillas, forros) y productos terminados. Contar con limitadas fuentes de estos recursos, con la falta de oferta de recurso humano calificado, la falta de acceso a tecnología $\mathrm{y}$, finalmente, dificultades de acceso a mercados y a fuentes de financiamiento, afecta su quehacer dentro del sector.

Con base en el contexto anterior, se formuló una investigación aplicada para identificar los elementos que impactan en la calidad de la producción del calzado escolar en El Salvador, a fin de proponer un estándar de calidad de referencia para el incremento de la misma y la competitividad de este rubro. Para ello se realizaron once pruebas de calidad en materias primas, componentes y producto terminado.

Como resultado de esto, se generó una propuesta de estándares de calidad para los productores y proveedores de materia prima, componente y producto terminado en la línea de calzado escolar. Esto facilitó las pruebas de validación de dichos estándares con la realización y análisis de ensayos en muestras nacionales de materia prima, componente y producto terminado. Esto permitió al sector involucrado un aseguramiento y mejora en la calidad de su producción; contribuyendo así a innovar e incrementar el valor agregado para la industria, permitiéndole volverse más competitivo.

Palabras clave: Calzado escolar, mediana empresa, pequeña empresa, microempresa, control de calidad, estandarización, norma técnica.

\begin{abstract}
Companies in the footwear industry in El Salvador, particularly micro, small and medium-sized companies, face situations that make them less competitive, such as: quality problems of raw materials (leather), components (soles, laces, insoles, linings) and finished products. Having limited sources of these resources, the lack of supply of qualified human resources, the lack of access to technology and, finally, difficulties in accessing markets and funding sources, affect their work within the sector.

With base on the above context, an applied research was formulated to identify the elements that impact the quality of the production of school footwear in El Salvador, in order to propose a reference quality standard for its increase and competitiveness of this item. For this, eleven quality tests were carried out on raw materials, components and finished product.

As a result, a proposal for quality standards was generated for producers and suppliers of raw materials, components and finished products in the line of school footwear. This facilitated the validation tests of these standards by conducting and analyzing trials on national samples of raw material, component and finished product. This allowed the sector involved to ensure and improve the quality of its production; Thus contributing to innovate and increase the added value for the industry, allowing it to become more competitive.
\end{abstract}

Key words: School footwear, medium Company, small company, micro company, quality control, standardization, technical standard.

1. Ingeniera Industrial, email: yaneth.gochez@catolica.edu.sv; ORCID: https://orcid.org/0000-0001-6506-8071

2. Ingeniera Industrial, email: rocio.retana@catolica.edu.sv; ORCID: https://orcid.org/0000-0002-3699-9982

3. Maestro en Dirección Estratégica de Empresas, email: alfredo.grande@catolica.edu.sv;

ORCID: https://orcid.org/0000-0003-2284-6990 


\section{Introducción}

La industria de calzado en El Salvador tiene una gran importancia por ser proveedora de un producto necesario que satisface una de las necesidades básicas del ser humano, siendo también una fuente generadora de empleo. Este sector se ha visto afectado por la participación significativa de productos importados considerados de baja calidad, provenientes -en su mayoría- de países asiáticos; y por la limitada producción nacional de materias primas, empleadas en la producción de estos bienes. Asimismo, no existen estándares nacionales como parámetro para contrastar la calidad necesaria de la producción de materias primas, y de esa forma volver más competitivo al sector. Todos los procesos en las micro, pequeñas y medianas empresas (MIPYMES) ${ }^{4}$ del rubro se realizan de forma empírica; no están regidos bajo normativas. A esto se suma que en El Salvador no existen escuelas especializadas que formen técnicos en calzado, ni laboratorios de control de calidad para materias primas, componentes y producto terminado.

En el marco del proyecto USAID de Educación Superior para el Crecimiento Económico, la Universidad Católica de El Salvador (UNICAES), junto a la Dirección de Innovación y Calidad del Ministerio de Economía (DICA/ MINEC) y la Cámara de la Industria del Calzado y Afines de El Salvador (CALZAES), se elaboró un plan para identificar los estándares nacionales existentes en el sector MIPYME de calzado en el país. A partir de esto, se buscó conocer los factores internos y externos que influyen en la calidad del mismo, mediante un estudio significativo de las pasantías realizadas en empresas, y el análisis comparativo de experiencias y normativas internacionales de calzado escolar de países como Perú, España y Colombia.

\section{Metodología}

La investigación de tipo experimental demandó la realización de una serie de ensayos de calidad dentro del Laboratorio de control de calidad para el calzado y afines, ubicado en la Universidad Católica de El Salvador (UNICAES). Esto con el fin de evaluar las materias primas e insumos empleados en el proceso de manufactura del calzado escolar por parte de la MIPYME del país; además de comparar dichos resultados con estándares internacionales que se manejan en países con condiciones similares. Finalmente, a partir de dicha comparación, se estableció una propuesta de norma de calidad para El Salvador.

Asimismo, se recopiló información mediante visitas de campo (observación) a quince empresas productoras, así como encuestas y entrevistas (cuestionarios) realizadas a los empresarios y al personal que laboraban dentro de ellas (un total de 45 personas, aproximadamente). La muestra de empresas que participaron en la investigación se seleccionó con el apoyo de la Dirección de Innovación y Calidad del Minis- 
terio de Economía. Debido a la naturaleza de la investigación, los empresarios debían proporcionar información confidencial de sus procesos y productos, encareciendo así la participación de la mayoría de ellos.

Para el trabajo de campo se contó con el apoyo de siete estudiantes de la carrera de Ingeniería Industrial: dos de ellos cursaban tercer año, $\mathrm{y}$ cinco se encontraban a nivel de cuarto año. Los criterios de selección para los alumnos que apoyaron el proyecto fueron los siguientes: ser estudiantes de la carrera antes mencionada, estar cursando o tener aprobada al menos una de las siguientes asignaturas: Ingeniería de Métodos, Control Estadístico de la Calidad y/o Administración de la Producción I.

Adicionalmente, se tuvo apoyo de los técnicos de la Dirección de Innovación y Calidad (DICA/MINEC) y empresarios de la Cámara de la Industria del Calzado y Afines de El Salvador (CALZAES) para el proceso de validación del trabajo realizado, a través de la facilitación de acceso a las instalaciones de las empresas y proveyendo información confidencial.

Las visitas de campo consistieron en pasantías de los investigadores y estudiantes dentro de las instalaciones de la empresa, con el objetivo de conocer el proceso de elaboración del calzado escolar, tanto masculino como femenino; además de evidenciar los recursos que se utilizan para el desarrollo de dicho proceso, tales como: maquinaria, materia prima, materiales indirectos, talento humano e información. Se realizaron las entrevistas a los empresarios y/o trabajadores antes, durante y después del recorrido de observación por la planta de producción, las oficinas, las bodegas y otras áreas dentro de la empresa. Los datos obtenidos se descargaron en un documento prediseñado con datos importantes que se deseaban levantar, debido a que no se contaba con una bibliografía de referencia de forma previa.

En resumen, la investigación se desarrolló a través de distintas fases:

- Fase uno: A través de un diagnóstico se identificó y priorizó los aspectos de calidad existentes en las MIPYMES de El Salvador, mediante estadías en empresas de interés ubicadas en diversos municipios del país: Santa Ana y Candelaria de La Frontera, correspondientes al departamento de Santa Ana; Santo Tomás, Mejicanos, San Salvador y Soyapango, pertenecientes al departamento de San Salvador; y Lourdes Colón, municipio del departamento de La Libertad.

- Fase dos: Se diseñó una propuesta de normativa de calidad para el calzado escolar, tanto en la materia prima, los componentes como en el producto terminado. Esta fase surgió del análisis de resultados de la fase 1 y de la comparación de estos con las prácticas actuales, que se vivencian en el sector proveedor de materiales y los productores de calzado.

- Fase tres: Se realizaron pruebas de laboratorio a diferentes muestras nacionales de materia prima, componentes y producto termi- 
nado, con el fin de conocer e identificar su acercamiento a los estándares internacionales de calidad.

- Fase cuatro: Se divulgaron los resultados obtenidos de las pruebas de laboratorio a las partes interesadas, entre ellos emprendedores del rubro calzado, proveedores y productores de materia prima, componente y fabricantes de calzado.

\section{Resultados}

\section{Fase uno}

A través de las visitas, la observación y las entrevistas realizadas se conocieron los factores internos y externos que influyen en la calidad del calzado (ver figura 1).

Algunos de los fabricantes de calzado realizan pruebas empíricas (ejemplo: la prueba de despegue del acabado de la piel, flexión suela, entre otros). El sector calzado en El Salvador no ha tenido un acercamiento con normalización y se desconocen los parámetros bajo norma de la materia prima que deben exigir a los provee- dores. Por consiguiente, los proveedores no se ven obligados a entregar hoja de especificaciones técnicas que asegure la calidad del producto a los fabricantes.

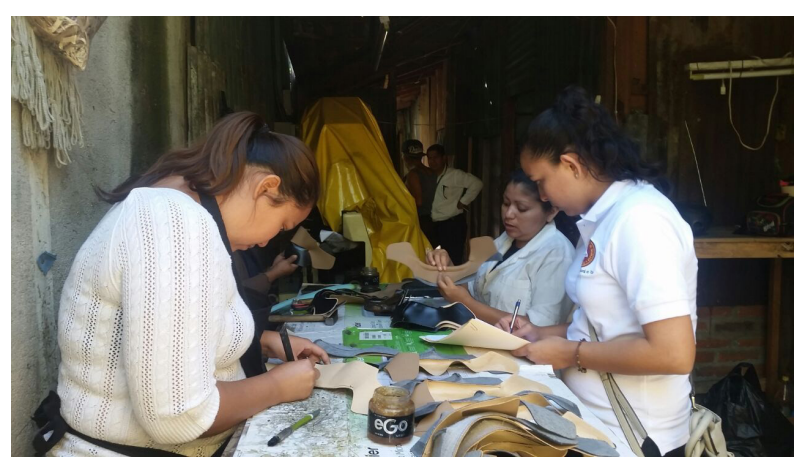

Figura 2. Estadía en empresa MIPYME del sector calzado.

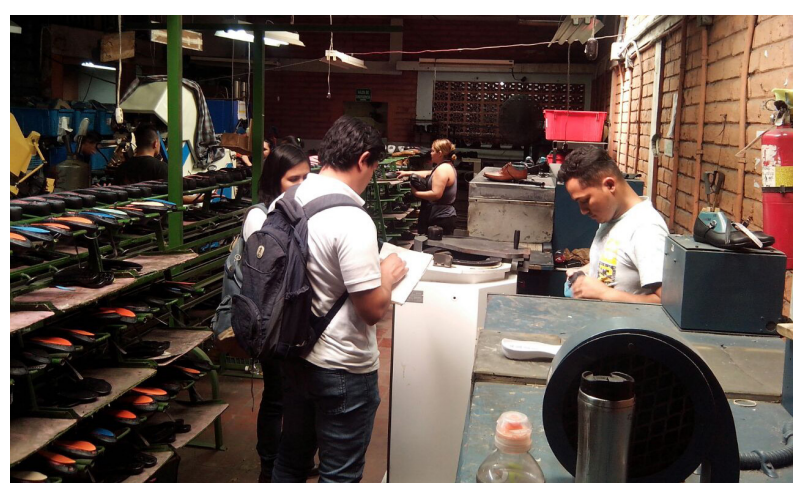

Figura 3. Estadía en empresa MIPYME del sector calzado.

\begin{tabular}{|l|l|}
\hline \multicolumn{1}{|c|}{ Factores internos } & \multicolumn{1}{c|}{ Factores externos } \\
\hline $\begin{array}{l}\text { Espacio físico: } \\
\text { - Infraestructura inadecuada }\end{array}$ & $\begin{array}{c}\text { Personal calificado: } \\
\text { - Poca formación académica }\end{array}$ \\
\hline $\begin{array}{l}\text { Manejo de insumos: } \\
\text { - Manejo deficiente de la materia prima }\end{array}$ & $\begin{array}{l}\text { Políticas estatales: } \\
\text { - Falta de acercamiento a la MIPYMES }\end{array}$ \\
\hline $\begin{array}{l}\text { Maquinaria y equipo: } \\
\text { - Inadecuada distribución }\end{array}$ & $\begin{array}{l}\text { Altos intereses: } \\
\text { - Falta de apoyo a la MIPYMES }\end{array}$ \\
\hline $\begin{array}{l}\text { Personal: } \\
\text { - No está comprometido con la empresa } \\
\text { - No tiene un método establecido de trabajo } \\
\text { - Personal poco calificado }\end{array}$ & $\begin{array}{l}\text { Materia prima ofertada: } \\
\text { - Mala calidad } \\
\text { Poca oferta }\end{array}$ \\
\hline
\end{tabular}

Figura 1. Factores internos y externos que influyen en la calidad del calzado. 
También se realizó una comparación entre las normas de países como Perú, España y Colombia, con el parámetro que se desea alcanzar como país. En función de esto, se presentan los ensayos para empeine o corte aparado (parte frontal-superior del calzado) (ver tabla 1).

En la tabla 1 se observan los parámetros referentes a las pruebas en cuero a ser utilizado en empeines o corte aparado. Esta comparación también se realizó para los parámetros correspondientes a suela, cordones, forro de cuero y textil; planta de montado (palmilla), plantilla y calzado terminado, con el fin de conocer todas las pruebas que se requieren por norma dentro de cada país, así como el estándar exigido. Esto evidencia que la norma peruana es la que posee los parámetros más accesibles para El Salvador.

Tabla 1. Ensayos para empeine o corte aparado

\begin{tabular}{|l|c|c|c|}
\cline { 2 - 4 } \multicolumn{1}{|c|}{ Ensayo } & Perú & España & Colombia \\
\hline $\begin{array}{l}\text { Resistencia a la flexión } \\
\text { en seco (mayor o igual, sin } \\
\text { daño apreciable) }\end{array}$ & 30 Kilociclos & 50 Kilociclos & 250 Kilociclos \\
\hline $\begin{array}{l}\text { Resistencia a la flexión en } \\
\text { húmedo (mayor o igual, sin } \\
\text { daño apreciable) }\end{array}$ & 10 Kilociclos & 12 Kilociclos & 50 Kilociclos \\
\hline $\begin{array}{l}\text { Resistencia al desgarro cue- } \\
\text { ro vacuno sin forro }\end{array}$ & $100 \mathrm{~N}$ & $100 \mathrm{~N}$ & $75 \mathrm{~N}$ \\
\hline $\begin{array}{l}\text { pH (acidez) sin forro (mayor } \\
\text { o igual) }\end{array}$ & $3.5 \mathrm{índice} \mathrm{de}$ & $3.5 \mathrm{índice}$ & 3.5 índice \\
\hline $\begin{array}{l}\text { Resistencia a la tracción } \\
\text { (mayor o igual) }\end{array}$ & $10 \mathrm{~N} / \mathrm{mm}^{2}$ & $20 \mathrm{~N} / \mathrm{mm}^{2}$ & de solidez \\
\hline $\begin{array}{l}\text { Permeabilidad al vapor de } \\
\text { agua (mayor o igual) }\end{array}$ & $0.8 \mathrm{mg} / \mathrm{cm}^{2} . \mathrm{h}$ & $1.5 \mathrm{mg} / \mathrm{cm}^{2} . \mathrm{h}$ & - \\
\hline $\begin{array}{l}\text { Solidez del color al frote } \\
\text { (mayor o igual) }\end{array}$ & $\geq 3 \mathrm{NEG}$ & $\geq 3 \mathrm{NEG}$ & - \\
\hline $\begin{array}{l}\text { Solidez del color al sudor } \\
\text { (mayor o igual) }\end{array}$ & $\geq 3 \mathrm{NEG}$ & $\geq 3 \mathrm{NEG}$ & - \\
\hline $\begin{array}{l}\text { Contenido de sustancias so- } \\
\text { lubles en agua }\end{array}$ & $3 \%$ & $1 \%$ & - \\
\hline
\end{tabular}




\section{Fase dos}

Durante este ciclo se definieron las especificaciones pertinentes de calidad para el calzado escolar en cuanto a materia prima, componentes y producto terminado. Esta etapa surgió del análisis de resultados de la fase anterior (fase 1), así como de la comparación de estos con las prácticas actuales que se vivencian en el sector proveedor de materiales y los productores de calzado.

De acuerdo a los resultados que se obtuvieron en las pasantías dentro de las empresas MIPYMES involucradas, respecto a la calidad de las materias primas, componentes y producto terminado, se concluyó que las pruebas de calidad realizadas son 100\% empíricas; es decir, que ninguna prueba es realizada dentro de un laboratorio de calidad para calzado. Debido a ello, se determinaron los ensayos imprescindibles a realizar durante la siguiente etapa (fase 3) para garantizar un producto de calidad en las MIPYMES del país.

Los ensayos a realizar y parámetros propuestos para el calzado escolar en materia prima, componentes y producto terminado se muestran en la tabla 2.

Tabla 2. Ensayos imprescindibles para materia prima, componente y producto terminado

\begin{tabular}{|c|c|c|c|c|c|}
\hline № & $\begin{array}{c}\text { Tipo } \\
\text { de ensayo }\end{array}$ & Definición del ensayo & $\begin{array}{c}\text { Propuesta } \\
\text { de parámetro }\end{array}$ & $\begin{array}{l}\text { Componente o } \\
\text { parte analizada }\end{array}$ & Equipo \\
\hline 1 & $\begin{array}{l}\text { Resistencia } \\
\text { de la unión } \\
\text { corte-piso } \\
\text { (suela) }\end{array}$ & $\begin{array}{l}\text { Determina la resistencia a la se- } \\
\text { paración del corte y el piso; o a la } \\
\text { separación de las capas adyacentes } \\
\text { del piso, o para producir el fallo por } \\
\text { desgarro del corte o del piso (suela). }\end{array}$ & $\geq 3 \mathrm{~N} / \mathrm{mm}$ & Calzado completo & $\begin{array}{l}\text { Máquina } \\
\text { universal }\end{array}$ \\
\hline 2 & $\begin{array}{l}\text { Resistencia } \\
\text { al desgarro } \\
\text { en corte } \\
\text { aparado o } \\
\text { empeine }\end{array}$ & $\begin{array}{l}\text { Determina la fuerza necesaria para } \\
\text { prolongar de manera progresiva, } \\
\text { hasta rotura de la incisión realizada } \\
\text { en la muestra. }\end{array}$ & $\geq 70 \mathrm{~N}$ & \multirow{3}{*}{$\begin{array}{c}\text { Empeine o corte } \\
\text { aparado }\end{array}$} & $\begin{array}{l}\text { Máquina } \\
\text { universal }\end{array}$ \\
\hline 3 & $\begin{array}{l}\text { Resistencia } \\
\text { a la flexión } \\
\text { en corte } \\
\text { aparado o } \\
\text { empeine }\end{array}$ & $\begin{array}{l}\text { Determina la resistencia a la flexión } \\
\text { del empeine, con dependencia del } \\
\text { material. Tiene la finalidad de evaluar } \\
\text { su aptitud para la utilización final. }\end{array}$ & 50,000 ciclos & & Flexómetro \\
\hline 4 & $\begin{array}{l}\text { Solidez del } \\
\text { color al fro- } \\
\text { te en cuero } \\
\text { aparado o } \\
\text { empeine }\end{array}$ & $\begin{array}{l}\text { Determina la resistencia de un ma- } \\
\text { terial a la decoloración y transferen- } \\
\text { cia del color de las superficies de los } \\
\text { materiales, durante un proceso de } \\
\text { abrasión en seco o en humedad. }\end{array}$ & $\begin{array}{c}\geq 3 \text { en escala } \\
\text { de grises }\end{array}$ & & Abrasímetro \\
\hline
\end{tabular}




\begin{tabular}{|c|c|c|c|c|c|}
\hline No & $\begin{array}{c}\text { Tipo } \\
\text { de ensayo } \\
\end{array}$ & Definición del ensayo & $\begin{array}{c}\text { Propuesta } \\
\text { de parámetro } \\
\end{array}$ & $\begin{array}{l}\text { Componente o } \\
\text { parte analizada }\end{array}$ & Equipo \\
\hline 5 & $\begin{array}{c}\text { Resistencia a } \\
\text { la abrasión } \\
\text { en forros }\end{array}$ & $\begin{array}{l}\text { Permite evaluar el desgaste en los } \\
\text { materiales que conforman los forros } \\
\text { y plantillas del calzado. }\end{array}$ & 25,600 ciclos & \multirow{3}{*}{ Forros } & \multirow{2}{*}{ Abrasímetro } \\
\hline 6 & $\begin{array}{l}\text { Solidez del } \\
\text { color al fro- } \\
\text { te en forros }\end{array}$ & $\begin{array}{l}\text { Determina el comportamiento de la } \\
\text { superficie del cuero al frote con fiel- } \\
\text { tro de lana. }\end{array}$ & $\begin{array}{c}\geq 3 \text { en escala } \\
\text { de grises }\end{array}$ & & \\
\hline 7 & $\begin{array}{l}\text { Resistencia } \\
\text { al desgarro } \\
\text { en forros }\end{array}$ & $\begin{array}{l}\text { Determina la fuerza necesaria para } \\
\text { prolongar de manera progresiva, } \\
\text { hasta rotura de la incisión realizada } \\
\text { en la muestra. }\end{array}$ & $\geq 15 \mathrm{~N}$ & & $\begin{array}{l}\text { Máquina } \\
\text { universal }\end{array}$ \\
\hline 8 & $\begin{array}{l}\text { Resistencia } \\
\text { a la flexión } \\
\text { en la suela }\end{array}$ & $\begin{array}{l}\text { Determina el número de flexiones } \\
\text { para provocar el aumento de una in- } \\
\text { cisión y/o la apariencia de grietas en } \\
\text { la suela. }\end{array}$ & $\begin{array}{l}\text { Crecimiento } \\
\text { de la incisión } \\
\geq 10 \mathrm{~mm}\end{array}$ & \multirow[b]{2}{*}{ Suela } & Flexómetro \\
\hline 9 & $\begin{array}{l}\text { Dureza en } \\
\text { la tapilla o } \\
\text { tacón }\end{array}$ & $\begin{array}{l}\text { Determina la dureza de indenta- } \\
\text { ción (dureza Shore) del elastóme- } \\
\text { tro vulcanizado o termoplástico } \\
\text { empleando. Durómetros con cuatro } \\
\text { tipos de escalas. }\end{array}$ & 80 Shore A & & Durómetro \\
\hline 10 & $\begin{array}{l}\text { Resistencia } \\
\text { a la abra- } \\
\text { sión en } \\
\text { cordones }\end{array}$ & $\begin{array}{l}\text { Determina la capacidad del cordón } \\
\text { para calzado de resistir al roce re- } \\
\text { petido contra un cordón similar o } \\
\text { un pasador. }\end{array}$ & 15,000 ciclos & \multirow{2}{*}{$\begin{array}{c}\text { Elementos de } \\
\text { cierre (cordones) }\end{array}$} & Abrasímetro \\
\hline 11 & $\begin{array}{l}\text { Resistencia } \\
\text { a la trac- } \\
\text { ción en los } \\
\text { cordones }\end{array}$ & $\begin{array}{l}\text { Determina la fuerza necesaria para } \\
\text { romper las pruebas a realizar por es- } \\
\text { tiramiento a velocidad constante. }\end{array}$ & $250 \mathrm{~N}$ & & $\begin{array}{l}\text { Máquina } \\
\text { universal }\end{array}$ \\
\hline 12 & $\begin{array}{l}\text { Absorción } \\
\text { y desorción } \\
\text { de agua en } \\
\text { la planta de } \\
\text { montado }\end{array}$ & $\begin{array}{l}\text { Determina la cantidad de vapor de } \\
\text { agua que absorbe un material en } \\
\text { un tiempo específico. Expresada en } \\
\text { masa de agua por unidad de superfi- } \\
\text { cie de material. }\end{array}$ & $\geq 70 \mathrm{mg} / \mathrm{cm}^{2}$ & $\begin{array}{c}\text { Planta } \\
\text { de montado }\end{array}$ & $\begin{array}{l}\text { Anali- } \\
\text { zador de } \\
\text { absorción y } \\
\text { desorción } \\
\text { de agua }\end{array}$ \\
\hline
\end{tabular}




\section{Fase tres}

Esta etapa consistió en desarrollar pruebas de calidad en laboratorio con diferentes muestras de materia prima, componentes y producto terminado de origen nacional, a fin de conocer e identificar su acercamiento a los estándares internacionales de calidad. Para ello, se adquirió el equipo necesario (máquina universal, flexómetro, frotómetro, durómetro, entre otros) que facilitó la realización de las pruebas.

Los resultados de estas pruebas permitieron conocer y analizar los parámetros actuales en los cuales se encuentra la fabricación del calzado escolar en la MIPYMES del país.

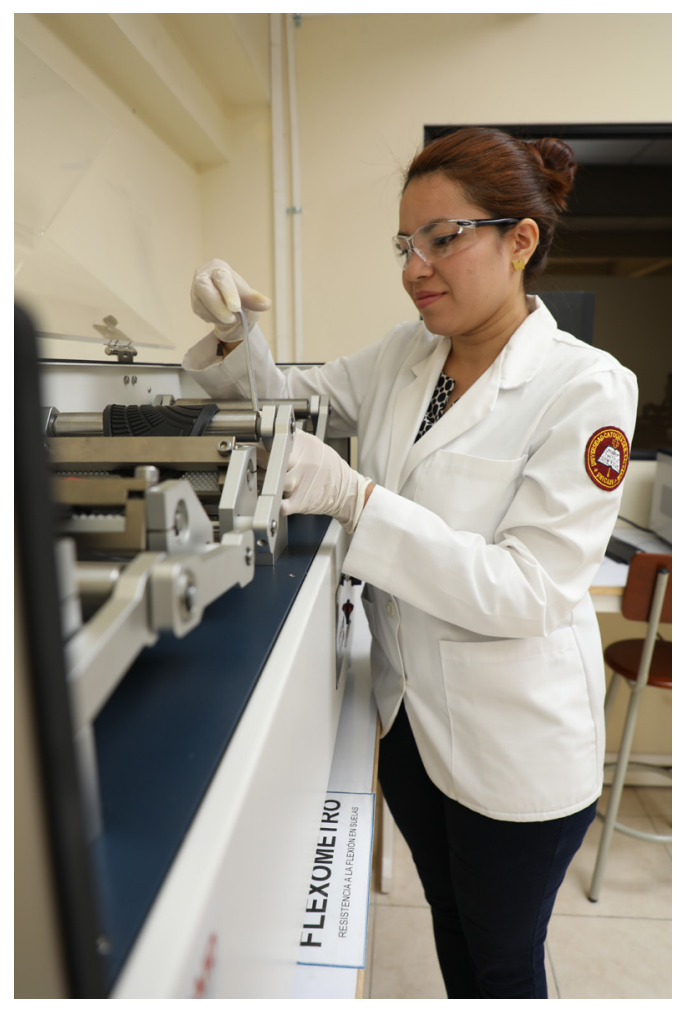

Figura 4. Ensayo de flexión en suela.

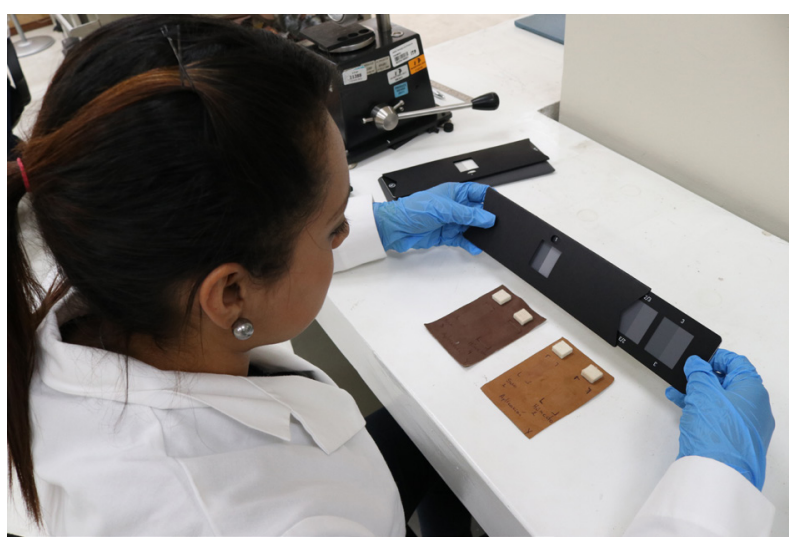

Figura 5. Ensayo de solidez del color al frote.

Para el análisis y evaluación de los parámetros de calidad en muestras de materiales y producto terminado, se realizaron cinco pruebas por ensayo. Las muestras de componente (suelas y cordones) fueron proporcionadas por empresarios productores de calzado. Todas las verificaciones se ejecutaron bajo las mismas condiciones ambientales, a una temperatura de $23^{\circ} \mathrm{C} \pm 2{ }^{\circ} \mathrm{C}$.

Para realizar las once pruebas en el laboratorio de control de calidad fueron indispensable los requerimientos mostrados en la tabla 3 .

Como resultado de la fase tres del proyecto, se validaron los resultados obtenidos mediante el equipo de laboratorio de calzado, teniendo la oportunidad de evaluar la calidad en el sector calzado del país. En la tabla 4, se muestran los resultados obtenidos en los once ensayos realizados y el parámetro internacional que le corresponde. 
Tabla 3. Requerimientos para las pruebas de control de calidad de materia prima, componentes y producto terminado del calzado escolar

\begin{tabular}{|c|c|c|c|c|c|c|}
\hline № & Prueba & $\begin{array}{l}\text { Norma de } \\
\text { referencia }\end{array}$ & $\begin{array}{c}\text { Ciclos } \\
\text { requeridos }\end{array}$ & Velocidad & $\begin{array}{c}\text { Equipo } \\
\text { utilizado }\end{array}$ & $\begin{array}{c}\text { Descripción de } \\
\text { la muestra }\end{array}$ \\
\hline 1 & $\begin{array}{l}\text { Resistencia } \\
\text { al despegue } \\
\text { de la unión } \\
\text { corte-piso }\end{array}$ & ISO 17708 & $\mathrm{~N} / \mathrm{A}$ & $\begin{array}{c}100 \\
\mathrm{~mm} / \mathrm{min}\end{array}$ & $\begin{array}{l}\text { Máquina } \\
\text { universal }\end{array}$ & $\begin{array}{l}\text { Pares de calzado } \\
\text { escolar termina- } \\
\text { do, color negro } \\
\text { para caballero. }\end{array}$ \\
\hline 2 & $\begin{array}{l}\text { Solidez del } \\
\text { color al frote } \\
\text { en cuero }\end{array}$ & ISO 17700 & $\begin{array}{c}100 \\
\text { (método } \\
\text { húmedo) }\end{array}$ & $\begin{array}{c}42 \\
\text { ciclos/min }\end{array}$ & Frotómetro & $\begin{array}{l}\text { Pieza de cuero } \\
\text { para forro en color } \\
\text { crema y marrón. }\end{array}$ \\
\hline 3 & $\begin{array}{l}\text { Resistencia } \\
\text { a la flexión } \\
\text { en cuero }\end{array}$ & ISO 17694 & 50,000 & $\begin{array}{c}100 \\
\text { ciclos/min }\end{array}$ & Flexómetro & $\begin{array}{l}\text { Piel obtenida de } \\
\text { la nuca, panza y } \\
\text { lomo de la res, } \\
\text { color negro. }\end{array}$ \\
\hline 4 & $\begin{array}{l}\text { Resistencia } \\
\text { a la abrasión } \\
\text { en cordones }\end{array}$ & ISO 22774 & 15,000 & $\begin{array}{c}60 \\
\text { ciclos/min }\end{array}$ & $\begin{array}{c}\text { Abrasíme- } \\
\text { tro }\end{array}$ & $\begin{array}{l}\text { Cordón negro para } \\
\text { calzado escolar, } \\
\text { con longitud de } \\
580 \mathrm{~mm} \text {. }\end{array}$ \\
\hline 5 & $\begin{array}{l}\text { Resistencia } \\
\text { a la tracción } \\
\text { en cordones }\end{array}$ & $\begin{array}{l}\text { UNE ISO } \\
59611\end{array}$ & $\mathrm{~N} / \mathrm{A}$ & $\begin{array}{c}100 \\
\mathrm{~mm} / \mathrm{min}\end{array}$ & $\begin{array}{l}\text { Máquina } \\
\text { universal }\end{array}$ & $\begin{array}{c}\text { Cordón negro para } \\
\text { calzado escolar. }\end{array}$ \\
\hline 6 & $\begin{array}{l}\text { Resistencia } \\
\text { a la abrasión } \\
\text { en forro textil }\end{array}$ & $\begin{array}{c}\text { ISO } 20344 \\
\text { apartado } \\
6.12\end{array}$ & 26,600 & 48 ciclos & $\begin{array}{c}\text { Abrasíme- } \\
\text { tro }\end{array}$ & $\begin{array}{l}\text { Pieza textil para } \\
\text { forro, color negro. }\end{array}$ \\
\hline 7 & $\begin{array}{c}\text { Dureza } \\
\text { en materiales } \\
\text { de suelas }\end{array}$ & $\begin{array}{l}\text { UNE-ISO } \\
7619-1\end{array}$ & $\mathrm{~N} / \mathrm{A}$ & $\mathrm{N} / \mathrm{A}$ & Durómetro & $\begin{array}{l}\text { Caucho color } \\
\text { negro y crema. }\end{array}$ \\
\hline
\end{tabular}




\begin{tabular}{|c|c|c|c|c|c|c|}
\hline № & Prueba & $\begin{array}{l}\text { Norma de } \\
\text { referencia }\end{array}$ & $\begin{array}{c}\text { Ciclos } \\
\text { requeridos }\end{array}$ & Velocidad & $\begin{array}{c}\text { Equipo } \\
\text { utilizado }\end{array}$ & $\begin{array}{c}\text { Descripción de } \\
\text { la muestra }\end{array}$ \\
\hline 8 & $\begin{array}{l}\text { Resistencia } \\
\text { a la flexión } \\
\text { en suela }\end{array}$ & ISO 17707 & 30,000 & $\begin{array}{c}100 \\
\text { ciclos/min }\end{array}$ & Flexómetro & $\begin{array}{c}\text { Suela negra } \\
\text { de PVC, para cal- } \\
\text { zado masculino, } \\
\text { talla } 42 .\end{array}$ \\
\hline 9 & $\begin{array}{l}\text { Absorción } \\
\text { y desorción } \\
\text { de agua en } \\
\text { la palmilla }\end{array}$ & $\begin{array}{l}\text { UNE ISO } \\
22649\end{array}$ & $\mathrm{~N} / \mathrm{A}$ & $\begin{array}{c}100 \\
\mathrm{~mm} / \mathrm{min}\end{array}$ & $\begin{array}{l}\text { Analizador } \\
\text { de absor- } \\
\text { ción y } \\
\text { desorción } \\
\text { de agua }\end{array}$ & $\begin{array}{l}\text { Cartón fibra de } \\
\text { espesor } 1.5 \mathrm{~mm} \text {, } \\
\text { color café. }\end{array}$ \\
\hline 10 & $\begin{array}{c}\text { Resistencia } \\
\text { al desgarro en } \\
\text { forro textil }\end{array}$ & ISO 13571 & $\mathrm{~N} / \mathrm{A}$ & $\begin{array}{c}100 \\
\mathrm{~mm} / \mathrm{min}\end{array}$ & $\begin{array}{l}\text { Máquina } \\
\text { universal }\end{array}$ & Pieza de forro \\
\hline 11 & $\begin{array}{c}\text { Resistencia } \\
\text { al desgarro } \\
\text { en cuero }\end{array}$ & ISO 13571 & $\mathrm{~N} / \mathrm{A}$ & $\begin{array}{c}100 \\
\mathrm{~mm} / \mathrm{min}\end{array}$ & $\begin{array}{l}\text { Máquina } \\
\text { universal }\end{array}$ & $\begin{array}{c}\text { Cuero negro } \\
\text { obtenido del lomo } \\
\text { de la res. }\end{array}$ \\
\hline
\end{tabular}

*Nota: Todas las normas de referencia son guías que indican el proceso (método, descripción de la probeta, maquinaria, equipo y materiales) para la realización de pruebas/ensayos físicos y químicos en materia prima, componentes y producto terminado, a fin de controlar la calidad del calzado escolar.

Las muestras analizadas en el laboratorio son un estadístico brinda la idea del estado actual de la calidad en el sector calzado. Los parámetros propuestos y validados mostrados en la tabla 4 son una base para procurar el cumplimiento de parámetros internacionales, mediante la mejorar continua.

\section{Fase cuatro}

Finalmente, se compartieron los resultados obtenidos, durante las diferentes fases. A los eventos realizados asistieron personas del sector calzado (emprendedores, proveedores y productores de materia prima, componente y fabricantes de calzado; empresarios MIPYMES, empresas ADOC e Industrias Caricia); así como representantes de USAID, Ministerio de Economía y Ministerio de Educación de El Salvador. 
Tabla 4. Propuesta validada de parámetros de calidad del calzado escolar

\begin{tabular}{|c|c|c|c|c|}
\hline № & Ensayo & Resultado & $\begin{array}{c}\text { Parámetro } \\
\text { Internacional }\end{array}$ & Propuesta validada \\
\hline 1 & $\begin{array}{c}\text { Resistencia a la abrasión } \\
\text { en cordones }\end{array}$ & 5191 ciclos & 15,000 ciclos & 10,000 ciclos \\
\hline 2 & $\begin{array}{l}\text { Resistencia de la unión } \\
\text { corte-piso }\end{array}$ & $1.2 \mathrm{~N} / \mathrm{mm}$ & $\geq 3 \mathrm{~N} / \mathrm{mm}$ & $\geq 2.5 \mathrm{~N} / \mathrm{mm}$ \\
\hline 3 & $\begin{array}{l}\text { Solidez de color al frote } \\
\text { en cuero }\end{array}$ & $\begin{array}{c}5 \text { en escala } \\
\text { de grises }\end{array}$ & $\begin{array}{c}\geq 3 \text { en escala de } \\
\text { grises }\end{array}$ & $\begin{array}{c}\geq 3 \text { en escala de } \\
\text { grises }\end{array}$ \\
\hline 4 & $\begin{array}{c}\text { Resistencia a la flexión } \\
\text { en cuero }\end{array}$ & $\begin{array}{l}50,000 \\
\text { ciclos }\end{array}$ & 50,000 ciclos & 50,000 ciclos \\
\hline 5 & $\begin{array}{c}\text { Resistencia a la tracción } \\
\text { en cordones }\end{array}$ & $213 \mathrm{~N}$ & $250 \mathrm{~N}$ & $250 \mathrm{~N}$ \\
\hline 6 & $\begin{array}{c}\text { Resistencia a la abrasión } \\
\text { en forro textil }\end{array}$ & $\begin{array}{l}25,600 \\
\text { ciclos }\end{array}$ & 25,600 ciclos & 51,200 ciclos \\
\hline 7 & $\begin{array}{c}\text { Determinación de la dureza } \\
\text { en tapilla o tacón }\end{array}$ & $\begin{array}{c}80.7 \\
\text { Shore A }\end{array}$ & 80 Shore A & 80 Shore A \\
\hline 8 & $\begin{array}{c}\text { Resistencia a la flexión } \\
\text { en suela }\end{array}$ & $0.0 \mathrm{~mm}$ & $\begin{array}{l}\text { Crecimiento de la } \\
\text { incisión } \leq 8 \mathrm{~mm}\end{array}$ & $\begin{array}{l}\text { Crecimiento de la } \\
\text { incisión } \leq 8 \mathrm{~mm}\end{array}$ \\
\hline 9 & $\begin{array}{l}\text { Análisis de absorción y } \\
\text { desorción de agua }\end{array}$ & $\begin{array}{c}112.93 \\
\mathrm{mg} / \mathrm{cm}^{2}\end{array}$ & $\geq 70 \mathrm{mg} / \mathrm{cm}^{2}$ & $\geq 70 \mathrm{mg} / \mathrm{cm}^{2}$ \\
\hline 10 & $\begin{array}{l}\text { Resistencia al desgarro } \\
\text { en forro textil }\end{array}$ & $27.44 \mathrm{~N}$ & $\geq 15 \mathrm{~N}$ & $\geq 15 \mathrm{~N}$ \\
\hline 11 & $\begin{array}{c}\text { Resistencia al desgarro } \\
\text { en cuero }\end{array}$ & $69.23 \mathrm{~N}$ & $\geq 70 \mathrm{~N}$ & $\geq 70 \mathrm{~N}$ \\
\hline
\end{tabular}

*Nota: Los datos colocados en la columna resultado corresponden al mejor valor obtenido en el ensayo.

\section{Discusión}

Las pruebas de ensayos de calidad realizadas en el laboratorio, con muestras de productores salvadoreños de materias primas, componentes y producto terminado tuvieron como propósito identificar los estándares nacionales existentes en el sector MIPYME de calzado en El Salva- dor. Gracias a los resultados se pudo establecer una propuesta de estándares de calidad que, como país, son alcanzables; considerando que estos se acoplan a normas internacionales.

Los estándares de calidad para las pruebas de ensayo de laboratorio que se consideran imprescindibles se muestran en la tabla 5. 
Tabla 5. Propuesta de parámetros de calidad a realizar en el calzado escolar de El Salvador

\begin{tabular}{|c|c|c|}
\hline Número & Ensayo & Parámetro salvadoreño propuesto \\
\hline 1 & Resistencia a la abrasión en cordones & 10,000 ciclos \\
\hline 2 & Resistencia de la unión corte-piso & $\geq 2.5 \mathrm{~N} / \mathrm{mm}$ \\
\hline 3 & Solidez de color al frote en cuero & $\geq 3$ en escala de grises \\
\hline 4 & Resistencia a la flexión en cuero & 50,000 ciclos \\
\hline 5 & Resistencia a la tracción en cordones & $250 \mathrm{~N}$ \\
\hline 6 & Resistencia a la abrasión en forro textil & 81,200 ciclos \\
\hline 7 & Determinación de la dureza en tapilla o tacón & $\mathrm{A}$ \\
\hline 8 & Resistencia a la flexión en suela & Crecimiento de la incisión $\leq 8 \mathrm{~mm}$ \\
\hline 9 & Análisis de absorción y desorción de agua & $\geq 70 \mathrm{mg} / \mathrm{cm}^{2}$ \\
\hline 10 & Resistencia al desgarro en forro textil & $\geq 15 \mathrm{~N}$ \\
\hline 11 & Resistencia al desgarro en cuero & $\mathrm{N}$ \\
\hline
\end{tabular}

De acuerdo a la propuesta de estándares, la materia prima, componentes y producto terminado deberán cumplir:

\section{Resistencia a la abrasión en cordones.}

Cada roce de cordón con cordón; o cordón con ojete (orificio donde se introducen los cordones o cintas en el calzado) es un ciclo cumplido. Se espera que la muestra no sufra rotura antes de los 10,000 ciclos.

2. Resistencia de la unión-corte piso. La fuerza ejercida para realizar el despegue debe ser mayor o igual a $2.5 \mathrm{~N} / \mathrm{mm}$.

\section{Solidez de color al frote en cuero para} empeine y forro. Deberá cumplir 150 ciclos (método seco) y 50 ciclos (método húmedo) de frote en estilo vaivén; teniendo una descarga de color en el fieltro y una degradación de color en el cuero, con una calificación mayor o igual a 3 en la escala de grises. (Esto sirve para determinar la solidez al color en cueros y forros sometidos a ensayo, evaluando la degradación y descarga del color, respectivamente).

4. Resistencia a la flexión en cuero. La muestra debe alcanzar 50,000 ciclos de flexiones, sin sufrir daños apreciables como, por ejemplo: grietas, arrugas o desprendimiento del acabado.

5. Resistencia a la tracción en cordones. $\mathrm{La}$ fuerza de rotura debe ser de $250 \mathrm{~N}$ mínimo para ser aceptable. Dentro de esta prueba, las roturas no deben estar cerca de las mordazas de la máquina universal al momento de realizarse.

\section{Resistencia a la abrasión en forro textil.} La muestra debe alcanzar 25,600 ciclos sin que sufra daños, es decir, sin que se generen agujeros en el forro.

\section{Determinación de la dureza en tapilla o} tacón. Al ejercer la presión por 15 segundos en el durómetro, la muestra debe marcar una dureza mayor o igual a $80^{\circ}$ Shore A. 
8. Resistencia a la flexión en suela. Al someterse la muestra a 30,000 flexiones, esta debe obtener un crecimiento de la incisión (corte en la línea de flexión de la suela) menor o igual $8 \mathrm{~mm}$.

9. Análisis de absorción y desorción de agua en la palmilla (planta de montado). La muestra analizada debe alcanzar un parámetro mayor o igual a $70 \mathrm{mg} / \mathrm{cm}^{2}$, con un flujo de agua (grado tres) de $7.5 \mathrm{ml} / \mathrm{min}$.

\section{Resistencia al desgarro en forro textil. $\mathrm{La}$} fuerza media necesaria para propagar el corte en la muestra debe ser mayor o igual a $15 \mathrm{~N}$.
11. Resistencia al desgarro en cuero. La fuerza media necesaria para propagar el corte en la muestra debe ser mayor o igual a $70 \mathrm{~N}$.

Con esta propuesta de estándares de calidad se genera una base de información que sirve para plantear acciones de mejora continua e innovación, en términos de procesos de normalización de las materias primas, componentes, procesos y productos terminados dentro de las MIPYMES del sector calzado.

\section{Referencias}

Asociación Española de Normalización y Certificación (1994). Footwear materials. Determinación de la resistencia a la tracción. UNE ISO 59-611-94. Madrid.

Asociación Española de Normalización y Certificación (2002). Calzado. Métodos de ensayo para empeines, forro y plantillas. Resistencia al desgarro. UNE-EN 13571. Madrid.

Asociación Española de Normalización y Certificación (2004). Calzado. Método de ensayo para accesorios: Cordones. Resistencia a la abrasión. UNE-EN ISO 22774. Madrid.

Asociación Española de Normalización y Certificación (2007). Calzado. Método de ensayo para empeines, forro y plantillas. Solidez del color al frote. UNE-EN ISO 17700. Madrid.

Asociación Española de Normalización y Certificación (2011). Caucho vulcanizado o termoplástico. Determinación de la dureza de indentación. Parte 1: Método del durómetro (dureza Shore). UNE-ISO 7619-1. Madrid.

Asociación Española de Normalización y Certificación (2012). Equipos de protección personal. Métodos de ensayo para calzado. UNE-EN ISO 20344. Madrid.

Asociación Española de Normalización y Certificación (2014). Calzado. Calzado de niño y colegial. Especificaciones y métodos de ensayo. UNE 59920. Madrid.

Asociación Española de Normalización y Certificación (2016). Calzado. Método de ensayo para empeines y forro. Resistencia a la flexión. UNE-EN ISO 17694. Madrid. 
Asociación Española de Normalización y Certificación (2016). Calzado. Métodos de ensayo palmillas y plantillas. Absorción y desorción de agua. UNE-EN ISO 22649. Madrid.

Banco Central de Reserva de El Salvador (noviembre 2016). Informe de Comercio Exterior de El Salvador enero - noviembre 2016. Recuperado de https://www.bcr.gob.sv/bcrsite/uploaded/ content/category/1311085361.pdf

ICONTEC (2017). Calzado de calle. Requisitos y métodos de ensayo. NTC 2038. Bogotá.

Instituto Nacional de Defensa de la Competencia y de la Protección de la Propiedad Intelectual (2014). Calzado. Calzado escolar. Requisitos y métodos de ensayo. NTP 241.0012014 . Perú.

Ministerio de Economía de El Salvador (2011). Informe Comercion Internacional. Primer Trimestre del 2011, (3). Recuperado de http://www.sice.oas.org/ctyindex/SLV/INFO_20111_s.pdf

Ministerio de Economía de El Salvador (2015). Perfil sectorial calzado 2015. Recuperado de http://dica.minec.gob.sv/attachments/article/825/Perfil\%20Sectorial\%20de\%20Calzado\%20 Dic\%20\%202015.pdf

Organismo Promotor de Exportaciones e Inversiones de El Salvador (2012). El Salvador un país de oportunidades en el sector calzado. Recuperado de http:/webcache.googleusercontent.com/search?q=cache:pwB_FuK5H0wJ:www.proesa.gob.sv/investment/documentation\%3Fdownload\%3D38:guia-de-calzado $\% 26$ start $\% 3 \mathrm{D} 20+\& \mathrm{~cd}=1 \& \mathrm{hl}=\mathrm{es} \& \mathrm{ct}=\mathrm{clnk} \& \mathrm{gl}=\mathrm{sv} \& \mathrm{client}=$ firefox-b-d

Organismo Salvadoreño de Normalización (2017). Calzado. Métodos de ensayo para suelas. Resistencia a la flexión. NTS ISO 17707. El Salvador.

Organismo Salvadoreño de Normalización (2017). Calzado. Métodos de ensayo para zapato completo. Resistencia de la unión corte-piso. NTC ISO 17708:2003. El Salvador 